\title{
NUMERICAL SOLUTION OF BLACK-SCHOLES OPTION PRICING WITH VARIABLE YIELD DISCRETE DIVIDEND PAYMENT
}

\author{
RAFAEL COMPANY, LUCAS JÓDAR and ENRIQUE PONSODA \\ Instituto de Matemática Multidisciplinar, Edificio $8 G$ \\ Universidad Politécnica de Valencia \\ Camino de Vera $s / n$, 46022 Valencia, Spain \\ E-mail: \{rcompany, ljodar, eponsoda\}@imm.upv.es
}

\begin{abstract}
This paper deals with the construction of numerical solution of the Black-Scholes (B-S) type equation modeling option pricing with variable yield discrete dividend payment at time $t_{d}$. Firstly the shifted delta generalized function $\delta\left(t-t_{d}\right)$ appearing in the B-S equation is approximated by an appropriate sequence of nice ordinary functions. Then a semidiscretization technique applied on the underlying asset is used to construct a numerical solution. The limit of this numerical solution is independent of the considered sequence of the nice type. Illustrative examples including the comparison with the exact solution recently given in [2] for the case of constant yield discrete dividend payment are presented.
\end{abstract}

1. Introduction. The Black-Scholes model for pricing stock options, when there are dividend payments $D(S, t)$, is

$$
\frac{\partial V}{\partial t}+\frac{1}{2} \sigma^{2} S^{2} \frac{\partial^{2} V}{\partial S^{2}}+(r S-D(S, t)) \frac{\partial V}{\partial S}-r V=0, \quad 0<S<\infty, \quad 0<t<T .
$$

If a discrete dividend payment with dividend date $t_{d}$ is considered, $D(S, t)$ takes the form

$$
D(S, t)=D_{\delta}(S) \delta\left(t-t_{d}\right), \quad 0<t_{d}<T,
$$

where $\frac{D_{\delta}(S)}{S}$ is the dividend yield and $\delta\left(t-t_{d}\right)$ is the shifted Dirac delta function (see [7, p. 140]). Recently, an explicit solution of (1) with a discrete dividend yield, independent of $S$, and a general payoff function $V(S, T)=f(S)$, has been given (see [2]).

2000 Mathematics Subject Classification: 03H10, 91B24, 35K15.

This work has been partially supported by the Generalitat Valenciana grant GVPRE/2008/ 092 and the Spanish M.C.Y.T. and FEDER grant TRA2007-68006-C02-02.

Key words and phrases: Black-Scholes equation, discrete dividends, variable yield, numerical solution, semidiscretization.

The paper is in final form and no version of it will be published elsewhere. 
This paper deals with the construction of numerical solutions of modified BlackScholes equation of the type

$$
\begin{gathered}
\frac{\partial V}{\partial t}+\frac{1}{2} \sigma^{2} S^{2} \frac{\partial^{2} V}{\partial S^{2}}+\left(r S-D_{\delta}(S) \delta\left(t-t_{d}\right)\right) \frac{\partial V}{\partial S}-r V=0, \\
V(S, T)=f(S), \quad 0<S<\infty, \quad 0<t_{d}<T, \quad 0<t<T .
\end{gathered}
$$

In order to guarantee that $S\left(t_{d}^{+}\right)$is not negative, a general realistic discrete dividend yield has the property that

$$
\int_{0}^{S\left(t_{d}^{-}\right)} \frac{d S}{D_{\delta}(S)}
$$

is infinite for any positive value of $S\left(t_{d}^{-}\right)$, see [7, p. 142].

This paper is organized as follows. Section 2 deals with preliminary results about the solution of the Black-Scholes equations without dividend payment as well as the approximation of the generalized function $\delta\left(t-t_{d}\right)$ by means of ordinary functions sequence $g_{n}(t)$. Furthermore, some previous results on matrix calculus are included.

Section 3 provides the numerical solution of the approximate problem

$$
\begin{gathered}
\frac{\partial V_{n}}{\partial t}+\frac{1}{2} \sigma^{2} S^{2} \frac{\partial^{2} V_{n}}{\partial S^{2}}+\left(r S-D_{\delta}(S) g_{n}(t)\right) \frac{\partial V_{n}}{\partial S}-r V_{n}=0 \\
0<S<\infty, \quad 0<t<T \\
V_{n}(S, T)=f(S), \quad 0<S<\infty,
\end{gathered}
$$

by a semidiscretization technique.

Finally, in section 4 , the numerical solution obtained in $t_{d}$ taking limits as $n$ tends to infinity, is extended to the interval $\left[0, t_{d}\right)$, and some illustrative examples are included.

2. Preliminaries. For clarity of presentation we recall some notation and results about the solution of the Black-Scholes equation without dividend payment as well as the concept and properties of the Dirac delta generalized function.

For $\eta, \nu \in R$, with $\eta<\nu$ we define the set $\mathcal{M}(\eta, \nu)$ as follows:

$$
\mathcal{M}(\eta, \nu)=\left\{f:(0, \infty) \rightarrow R\left|\int_{0}^{\infty} x^{\alpha-1}\right| f(x) \mid d x<\infty, \quad \eta<\alpha<\nu\right\} .
$$

If there exists $\eta<\nu$ such that $f \in \mathcal{M}(\eta, \nu)$, then the solution of the Black-Scholes equation

$$
\left.\begin{array}{c}
\frac{\partial V_{B S}}{\partial t}+\frac{1}{2} \sigma^{2} S^{2} \frac{\partial^{2} V_{B S}}{\partial S^{2}}+r S \frac{\partial V_{B S}}{\partial S}-r V_{B S}=0, \\
V_{B S}(S, T)=f(S), \quad 0<S<\infty, \quad 0<t<T,
\end{array}\right\}
$$

is given by

$$
V_{B S}(S, t)=\frac{e^{-r(T-t)}}{\sigma \sqrt{2 \pi(T-t)}} \int_{-\infty}^{\infty} f\left(e^{-l}\right) e^{-\frac{\left[\ln S+l+(T-t)\left(r-\frac{\sigma^{2}}{2}\right)\right]^{2}}{2 \sigma^{2}(T-t)}} d l
$$

(see [1]). 
We denote by $K$ the space of functions $\varphi: R \rightarrow R$ in $C^{\infty}(R)$ having a compact support. A generalized function $g$ is defined as a continuous linear functional on $K$, and we denote $g(\varphi)=(g, \varphi)$ (see [5, p. 11]). The space of all generalized functions on $K$ will be called $K^{\prime}$. The Dirac delta function is defined as the generalized function which assigns value $\varphi(0)$ to each function $\varphi(x) \in K$, i.e., $(\delta, \varphi)=\varphi(0)$. Note that the shifted Dirac delta function $\delta\left(t-t_{d}\right)$ acts on $K$ in the form $\left(\delta\left(t-t_{d}\right), \varphi(t)\right)=\varphi\left(t_{d}\right)$, see [5, pp. 11-13].

A sequence of ordinary functions $\left\{g_{n}(t)\right\}$ converges in $K^{\prime}$ to the generalized function $g$ if for all $\varphi \in K($ see $[5$, p. 63]),

$$
(g, \varphi)=\lim _{n \rightarrow \infty}\left(g_{n}, \varphi\right)=\lim _{n \rightarrow \infty} \int_{-\infty}^{+\infty} g_{n}(t) \varphi(t) d t .
$$

Definition 1. A sequence of ordinary functions $\left\{g_{n}(t)\right\}$ is said to be a very nice shifted delta-defining sequence if for each $n \geq 0, g_{n}(t)$ has support $\left[t_{d}-\frac{1}{2 n}, t_{d}+\frac{1}{2 n}\right]$, and is a continuous nonnegative function that satisfies

$$
\int_{t_{d}-\frac{1}{2 n}}^{t_{d}+\frac{1}{2 n}} g_{n}(t) d t=1
$$

Taking into account [5, p. 65], a very nice shifted delta-defining sequence converges in $K^{\prime}$ to the generalized function $\delta\left(t-t_{d}\right)$. Concrete examples of such sequences may be found in $[5$, p. 66].

Troughout this paper, $\|y\|_{2}$ denotes the usual Euclidean norm of a vector $y$. If $A$ is a matrix in $C^{p \times p}$, its two-norm, denoted by $\|A\|$ is defined as

$$
\|A\|=\max \left\{+\sqrt{\lambda} ; \quad \lambda \in \sigma\left(A^{H} A\right)\right\}
$$

where $A^{H}$ denotes the transconjugate of $A$ and $\sigma(M)$ is the set of all eigenvalues of a matrix $M \in C^{p \times p}$.

If $A$ is a matrix in $C^{p \times p}$, then

$$
\left\|e^{t A}\right\| \leq e^{t \mu(A)}, \quad t \geq 0
$$

where $\mu(A)$ is the logarithmic norm of $A$, defined by

$$
\mu(A)=\max \left\{\lambda ; \quad \lambda \in \sigma\left(\frac{A+A^{H}}{2}\right)\right\} .
$$

From [3, p. 110], [4], if $A$ and $B$ are matrices in $C^{p \times p}$, the following properties hold true:

$$
\begin{gathered}
\mu(A+B) \leq \mu(A)+\mu(B), \\
|\mu(A)| \leq\|A\|, \\
\mu(c A)=|c| \mu(\operatorname{sgn}(c) A), \quad c \in R .
\end{gathered}
$$

By [3, p. 112], the solution of the linear system

$$
X^{\prime}(t)=P(t) X(t)+b(t), \quad X(0)=X_{0} \in C^{m}, \quad t \geq 0,
$$

satisfies

$$
\|X(t)\| \leq\left\|X_{0}\right\| e^{\int_{0}^{t} \mu(P(s)) d s}+\int_{0}^{t} e^{\int_{v}^{t} \mu(P(z)) d z}\|b(v)\|_{2} d v,
$$

where $P(t)$ and $b(t)$ are continuous functions taking values in $C^{m \times m}$ and $C^{m}$ respectively. 
3. Numerical solution of the approximate problem. Let us consider problem (5) where $\left\{g_{n}(t)\right\}$ is an arbitrary very nice shifted delta defining sequence. Taking into account Definition 1, for $t_{d}+\frac{1}{2 n}<t<T$ one gets

$$
\left.\begin{array}{c}
\frac{\partial V_{n}}{\partial t}+\frac{1}{2} \sigma^{2} S^{2} \frac{\partial^{2} V_{n}}{\partial S^{2}}+r S \frac{\partial V_{n}}{\partial S}-r V_{n}=0, \\
V_{n}(S, T)=f(S), \quad 0<S<\infty, \quad t_{d}+\frac{1}{2 n}<t<T,
\end{array}\right\}
$$

that is, the Black-Scholes equation (6). Hence, the solution of (5) in the interval $\left[t_{d}+\frac{1}{2 n}, T[\right.$ is given by

$$
V_{n}(S, t)=V_{B S}(S, t), \quad t_{d}+\frac{1}{2 n} \leq t<T .
$$

Then, problem (5) in the interval $t_{d}-\frac{1}{2 n} \leq t<t_{d}+\frac{1}{2 n}$ can be written by

$$
\left.\begin{array}{c}
\frac{\partial V_{n}}{\partial t}+\frac{1}{2} \sigma^{2} S^{2} \frac{\partial^{2} V_{n}}{\partial S^{2}}+\left(r S-D_{\delta}(S) g_{n}(t)\right) \frac{\partial V_{n}}{\partial S}-r V_{n}=0, \\
V_{n}\left(S, t_{d}+\frac{1}{2 n}\right)=V_{B S}\left(S, t_{d}+\frac{1}{2 n}\right), \quad 0<S<\infty, \quad t_{d}-\frac{1}{2 n} \leq t<t_{d}+\frac{1}{2 n} .
\end{array}\right\}
$$

We propose a semidiscretization method, see [6, p. 111], for solving (16). Let us consider an interval $[a, b]$ and $h$, an increment of $S, 0<a \leq S \leq b$, where $b-a=h N, S_{j}=a+j h$, $j=0,1, \ldots, N$. We introduce the notation $v_{n j}(t) \approx V_{n}\left(S_{j}, t\right)$ for $j=0,1, \ldots, N$. Then we replace the partial derivatives by finite expressions of the form

$$
\begin{gathered}
\frac{\partial V_{n}}{\partial S}\left(S_{j}, t\right) \approx \frac{v_{n j+1}(t)-v_{n j-1}(t)}{2 h}, \quad j=1, \ldots, N-1, \\
\frac{\partial^{2} V_{n}}{\partial S^{2}}\left(S_{j}, t\right) \approx \frac{v_{n j+1}(t)-2 v_{n j}(t)+v_{n j-1}(t)}{h^{2}}, j=1, \ldots, N-1,
\end{gathered}
$$

From (16), (17) and (18), one gets

$$
\left.\begin{array}{c}
\frac{d v_{n j}(t)}{d t}=\alpha_{j} v_{n j-1}(t)+\beta_{j} v_{n j}(t)+\gamma_{j} v_{n j+1}(t)-\frac{d_{j} g_{n}(t)}{2 h}\left(v_{n j-1}(t)-v_{n j+1}(t)\right), \\
j=1, \ldots, N-1,
\end{array}\right\}
$$

where

$$
\left.\begin{array}{l}
d_{j}=D_{\delta}\left(S_{j}\right) \\
\alpha_{j}=-\frac{1}{2} \sigma^{2} \frac{S_{j}^{2}}{h^{2}}+r \frac{S_{j}}{2 h} \\
\beta_{j}=\frac{\sigma^{2} S_{j}^{2}}{h^{2}}+r \\
\gamma_{j}=-\frac{1}{2} \sigma^{2} \frac{S_{j}^{2}}{h^{2}}-r \frac{S_{j}}{2 h}
\end{array}\right\}
$$

In order to link the boundary values $v_{n 0}(t)$ and $v_{n N}(t)$ with the rest of the solution, let us 
assume a quadratic approximation given by interpolation Lagrange polynomial of second degree to obtain auxiliary values $v_{n-1}(t)$ and $v_{n N+1}(t)$.

Taking into account

$$
P(S)=\frac{\left(S-S_{1}\right)\left(S-S_{2}\right)}{\left(S_{0}-S_{1}\right)\left(S_{0}-S_{2}\right)} v_{n 0}(t)+\frac{\left(S-S_{0}\right)\left(S-S_{2}\right)}{\left(S_{1}-S_{0}\right)\left(S_{1}-S_{2}\right)} v_{n 1}(t)+\frac{\left(S-S_{0}\right)\left(S-S_{1}\right)}{\left(S_{2}-S_{0}\right)\left(S_{2}-S_{1}\right)} v_{n 2}(t)
$$

one gets for $S_{-1}=S_{0}-h$

$$
v_{n-1}(t)=3 v_{n 0}(t)-3 v_{n 1}(t)+v_{n 2}(t),
$$

and assuming equation (19) for $j=0$, it follows that

$$
\begin{aligned}
\frac{d v_{n 0}(t)}{d t}= & \left(3 \alpha_{0}+\beta_{0}\right) v_{n 0}(t)+\left(-3 \alpha_{0}+\gamma_{0}\right) v_{n 1}(t)+\alpha_{0} v_{n 2}(t) \\
& -\frac{d_{0} g_{n}(t)}{2 h}\left(3 v_{n 0}(t)-4 v_{n 1}(t)+v_{n 2}(t)\right),
\end{aligned}
$$

where $d_{0}, \alpha_{0}, \beta_{0}$ and $\gamma_{0}$ are given by (20) for $j=0$.

Similarly one gets for $v_{n N}(t)$ the equation

$$
\begin{aligned}
\frac{d v_{n N}(t)}{d t}= & \gamma_{N} v_{n N-2}(t)+\left(\alpha_{N}-3 \gamma_{N}\right) v_{n N-1}(t)+\left(\beta_{N}+3 \gamma_{N}\right) v_{n N}(t) \\
& -\frac{d_{N} g_{n}(t)}{2 h}\left(-v_{n N-2}(t)+4 v_{n N-1}(t)-3 v_{n N}(t)\right)
\end{aligned}
$$

where $d_{N}, \alpha_{N}, \beta_{N}$ and $\gamma_{N}$ are given by (20) for $j=N$.

Let us denote

$$
v_{n}(t)=\left[\begin{array}{c}
v_{n 0}(t) \\
v_{n 1}(t) \\
\vdots \\
v_{n N}(t)
\end{array}\right] \in R^{(N+1) \times 1} .
$$

Taking into account equations (19), (22) and (23), one gets

$$
\frac{d v_{n}(t)}{d t}=\left(M-g_{n}(t) B\right) v_{n}(t), \quad t_{d}-\frac{1}{2 n} \leq t<t_{d}+\frac{1}{2 n},
$$

where

$$
M=\left[\begin{array}{ccccccccc}
3 \alpha_{0}+\beta_{0} & -3 \alpha_{0}+\gamma_{0} & \alpha_{0} & 0 & 0 & \cdots & 0 & 0 & 0 \\
\alpha_{1} & \beta_{1} & \gamma_{1} & 0 & 0 & \cdots & 0 & 0 & 0 \\
0 & \alpha_{2} & \beta_{2} & \gamma_{2} & 0 & \cdots & 0 & 0 & 0 \\
\vdots & \vdots & \ddots & \ddots & \ddots & & \vdots & \vdots & \vdots \\
& & & & & & & & \\
0 & \vdots & \vdots & \vdots & & \ddots & \ddots & \ddots & \vdots \\
0 & 0 & 0 & 0 & 0 & \cdots & \alpha_{N-1} & \beta_{N-1} & \gamma_{N-1} \\
& 0 & 0 & 0 & 0 & \cdots & \gamma_{N} & \alpha_{N}-3 \gamma_{N} & \beta_{N}+3 \gamma_{N}
\end{array}\right]
$$




$$
B=\frac{1}{2 h}\left[\begin{array}{ccccccccc}
3 d_{0} & -4 d_{0} & d_{0} & 0 & 0 & \cdots & 0 & 0 & 0 \\
d_{1} & 0 & -d_{1} & 0 & 0 & \cdots & 0 & 0 & 0 \\
0 & d_{2} & 0 & -d_{2} & 0 & \cdots & 0 & 0 & 0 \\
\vdots & \vdots & \ddots & \ddots & \ddots & & \vdots & \vdots & \vdots \\
& & & & & & & & \\
\vdots & \vdots & \vdots & \vdots & & \ddots & \ddots & \ddots & \vdots \\
0 & 0 & 0 & 0 & 0 & \cdots & d_{N-1} & 0 & -d_{N-1} \\
0 & 0 & 0 & 0 & 0 & \cdots & -d_{N} & 4 d_{N} & -3 d_{N}
\end{array}\right] .
$$

Note that $M$ and $B$ depend on $S_{j}$ and $h$ but not on $t$. The solution vector $v_{n}(t)$ must satisfy the final condition

$$
v_{n}\left(t_{d}+\frac{1}{2 n}\right)=\left[\begin{array}{c}
V_{B S}\left(S_{0}, t_{d}+\frac{1}{2 n}\right) \\
\vdots \\
V_{B S}\left(S_{N}, t_{d}+\frac{1}{2 n}\right)
\end{array}\right] \in R^{(N+1) \times 1} .
$$

For problem (25)-(28) we do not have an explicit formula for the solution if $M$ and $B$ are arbitrary noncommuting matrices. However, we are not interested in the explicit solution but in the limit

$$
v\left(t_{d}^{-}\right)=\lim _{n \rightarrow \infty} v_{n}\left(t_{d}-\frac{1}{2 n}\right) .
$$

Let us consider firstly the problem

$$
\begin{aligned}
\frac{d \widetilde{v}_{n}(t)}{d t}= & -g_{n}(t) B \widetilde{v}_{n}(t), \quad t_{d}-\frac{1}{2 n} \leq t<t_{d}+\frac{1}{2 n} \\
& \widetilde{v}_{n}\left(t_{d}+\frac{1}{2 n}\right)=v_{n}\left(t_{d}+\frac{1}{2 n}\right)
\end{aligned}
$$

The solution of (30) is given by

$$
\widetilde{v}_{n}(t)=e^{-\int_{t_{d}+1 / 2 n}^{t} g_{n}(s) d s B} v_{n}\left(t_{d}+\frac{1}{2 n}\right) .
$$

Taking into account Definition 1, one gets

$$
\widetilde{v}_{n}\left(t_{d}-\frac{1}{2 n}\right)=e^{B} v_{n}\left(t_{d}+\frac{1}{2 n}\right)
$$

and hence

$$
\widetilde{v}\left(t_{d}^{-}\right)=\lim _{n \rightarrow \infty} \widetilde{v}_{n}\left(t_{d}-\frac{1}{2 n}\right)=e^{B} v\left(t_{d}^{+}\right),
$$

where

$$
v\left(t_{d}^{+}\right)=\left[\begin{array}{c}
V_{B S}\left(S_{0}, t_{d}\right) \\
\vdots \\
V_{B S}\left(S_{N}, t_{d}\right)
\end{array}\right]
$$

In order to prove

$$
v\left(t_{d}^{-}\right)=\widetilde{v}\left(t_{d}^{-}\right) .
$$


let us denote the error vector $\varphi_{n}(t)$ such that

$$
v_{n}(t)=\widetilde{v}_{n}(t)+\varphi_{n}(t) .
$$

It is easy to check that $\varphi_{n}(t)$ satisfies the differential equation

$$
\left.\begin{array}{c}
\frac{d \varphi_{n}(t)}{d t}=\left(M-g_{n}(t) B\right) \varphi_{n}(t)+M \widetilde{v}_{n}(t), \\
\varphi_{n}\left(t_{d}+\frac{1}{2 n}\right)=0, \quad t_{d}-\frac{1}{2 n} \leq t<t_{d}+\frac{1}{2 n} .
\end{array}\right\}
$$

Taking into account the substitution $\tau=t_{d}+\frac{1}{2 n}-t$ and denoting

$$
\begin{aligned}
& \Psi_{n}(\tau)=\varphi_{n}\left(t_{d}+\frac{1}{2 n}-\tau\right), \\
& h_{n}(\tau)=g_{n}\left(t_{d}+\frac{1}{2 n}-\tau\right), \\
& \widetilde{w}_{n}(\tau)=\widetilde{v}_{n}\left(t_{d}+\frac{1}{2 n}-\tau\right),
\end{aligned}
$$

problem (37) can be written in the form

$$
\begin{gathered}
\frac{d \Psi_{n}(\tau)}{d \tau}=\left(-M+h_{n}(\tau) B\right) \Psi_{n}(\tau)-M \widetilde{w}_{n}(\tau) \\
\Psi_{n}(0)=0, \quad 0 \leq \tau \leq \frac{1}{n}
\end{gathered}
$$

From (14) and (38) it follows that

$$
\left\|\Psi_{n}(\tau)\right\|_{2} \leq \int_{0}^{\tau} e^{\int_{s}^{\tau} \mu\left(-M+h_{n}(z) B\right) d z}\|M\|\left\|\widetilde{w}_{n}(s)\right\|_{2} d s .
$$

By (11), (13) and taking into account Definition 1 and (39), one gets

$$
\left\|\Psi_{n}\left(\frac{1}{n}\right)\right\|_{2} \leq e^{|\mu(-M)| / n+|\mu(B)|}\|M\| \int_{0}^{\frac{1}{n}}\left\|\widetilde{w}_{n}(s)\right\|_{2} d s .
$$

From (12), (31), (37), (38) and (40) it follows that

$$
\left\|\Psi_{n}\left(\frac{1}{n}\right)\right\|_{2} \leq k\|M\| e^{\|M\| / n+\|B\|} \int_{0}^{\frac{1}{n}}\left\|e^{B \int_{0}^{s} h_{n}(z) d z}\right\| d s,
$$

where

$$
k=\max _{t_{d} \leq t \leq T}\left\{\left\|\left(V_{B S}\left(S_{0}, t\right), \ldots, V_{B S}\left(S_{N}, t\right)\right)\right\|\right\} .
$$

Finally, taking into account (9) and (13), one gets

$$
\left\|\Psi_{n}\left(\frac{1}{n}\right)\right\|_{2} \leq k\|M\| e^{\|M\| / n+\|B\|} \int_{0}^{\frac{1}{n}} e^{\mu(B)} d s \leq k\|M\| e^{\|M\|+2\|B\|} \frac{1}{n} .
$$

We have proved that

$$
\lim _{n \rightarrow \infty}\left\|\Psi_{n}\left(\frac{1}{n}\right)\right\|_{2}=0
$$


and by (33), (36), (37), one gets

$$
\begin{gathered}
\lim _{n \rightarrow \infty}\left\|v_{n}\left(t_{d}-\frac{1}{2 n}\right)-e^{B} v\left(t_{d}^{+}\right)\right\|_{2} \\
\leq \lim _{n \rightarrow \infty}\left\|v_{n}\left(t_{d}-\frac{1}{2 n}\right)-\widetilde{v}_{n}\left(t_{d}-\frac{1}{2 n}\right)\right\|_{2}+\lim _{n \rightarrow \infty}\left\|\widetilde{v}_{n}\left(t_{d}-\frac{1}{2 n}\right)-e^{B} v\left(t_{d}^{+}\right)\right\|_{2}=0 .
\end{gathered}
$$

Then the following result has been established.

THEOREM 1. Let $\left\{g_{n}(t)\right\}$ be a very nice shifted delta defining sequence given by Definition 1. Then the sequence of solutions $\left\{v_{n}\left(t_{d}-\frac{1}{2 n}\right)\right\}$ of the problems (25)-(28) converges to

$$
v\left(t_{d}^{-}\right)=e^{B} v\left(t_{d}^{+}\right)
$$

where $B$ and $v\left(t_{d}^{+}\right)$are defined by (27) and (34) respectively.

4. Prolongation of the solution to $\left[0, t_{d}\right)$ and examples. In order to construct the numerical solution of (3)-(4) in the interval $\left[0, t_{d}\right)$, we use also the semidiscretization method proposed in section 3. Taking into account (41) and the differential system (25) for $g_{n}(t)=0$, one gets

$$
\left.\begin{array}{c}
\frac{d v(t)}{d t}=M v(t), \quad 0 \leq t<t_{d}, \\
v\left(t_{d}\right)=e^{B} v\left(t_{d}^{+}\right),
\end{array}\right\}
$$

where $M$ is defined by (26). Solving (42) it follows that

$$
v(t)=e^{-M\left(t_{d}-t\right)} e^{B} v\left(t_{d}^{+}\right), \quad 0 \leq t<t_{d} .
$$

The following example compares the exact solution of the valuation of a vanilla call option with constant yield discrete dividend payment with the numerical solution constructed using the previous approach.

EXAMPLE 1. Let us consider the valuation problem of a call option with discrete dividend, modeled by (3)-(4), where

$$
D_{\delta}(S)=A \delta\left(t-t_{d}\right) S
$$

and

$$
f(S)=\max \{S-E, 0\}, \quad 0<S<\infty .
$$

In this case the discrete dividend payment has got a constant dividend yield $A$. The solution of (44)-(45) is given by (see [2])

$$
V(S, t)= \begin{cases}S e^{-A} N\left(d_{1}^{\prime}\right)-E e^{-r(T-t)} N\left(d_{2}^{\prime}\right), & 0<t<t_{d}, \\ S N\left(d_{1}\right)-E e^{-r(T-t)} N\left(d_{2}\right), & t_{d}<t<T,\end{cases}
$$

where

$$
d_{1}=\frac{1}{\sigma \sqrt{T-t}}\left[\ln \frac{S}{E}+(T-t)\left(r+\frac{\sigma^{2}}{2}\right)\right]
$$




$$
\begin{gathered}
d_{2}=\frac{1}{\sigma \sqrt{T-t}}\left[\ln \frac{S}{E}+(T-t)\left(r-\frac{\sigma^{2}}{2}\right)\right] \\
d_{i}^{\prime}=d_{i}-\frac{A}{\sigma \sqrt{T-t}}, \quad i=1,2,
\end{gathered}
$$

and

$$
N(x)=\frac{1}{\sqrt{2 \pi}} \int_{-\infty}^{x} e^{-\frac{\xi^{2}}{2}} d \xi,
$$

is the cumulative probability function for a standardized normal variable.

For $A=0.1, \sigma=0.1, r=0.08, T=1, t_{d}=0.5, E=7, S_{0}=3, N=34$ and $h=0.5$, one gets for the value at $t=0$ the mean error

$$
\frac{\|v(0)-V(0)\|}{N+1}=0.000459,
$$

where $v(0)$ is obtained by using $(43)$ and $V(0)$ is the vector of the exact solutions given by (46) valuating at grid points $S_{i}, 0 \leq i \leq 34$, and time $t=0$.

The continuous line, denoted by dividend type $C$, in figure 1 is the numerical valuation of this call option at $t=0$ for $N=100$.

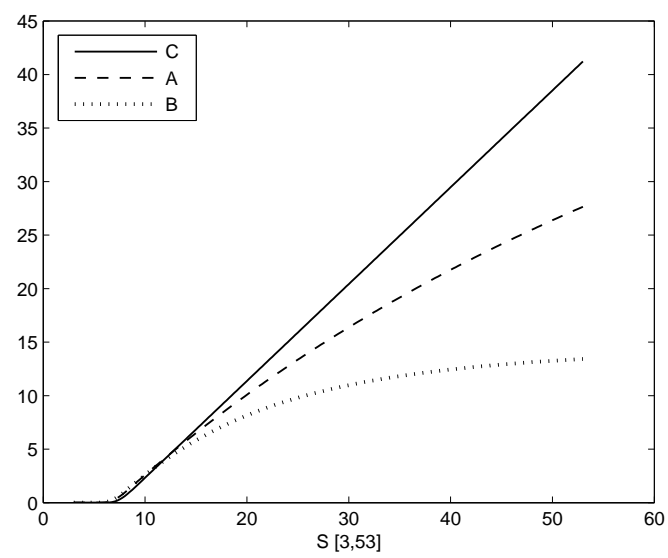

Fig. 1. Call options with strike price $\mathrm{E}$ and different dividend payments

The following examples deal with the application of the numerical method to variable yield discrete dividend payment cases.

EXAMPLE 2. Let us consider the valuation problem of a vanilla call option modeled by (3)-(4), where

$$
D_{\delta}(S)=A S^{2}
$$

and

$$
f(S)=\max \{S-E, 0\}, \quad 0<S<\infty .
$$

For $A=0.01, \sigma=0.1, r=0.08, T=1, t_{d}=0.5, E=7, S_{0}=3, N=100$ and $h=0.5$, by (43) one gets the numerical solution $v(0)$, see the dashed line in figure 1 , called dividend type $A$. 


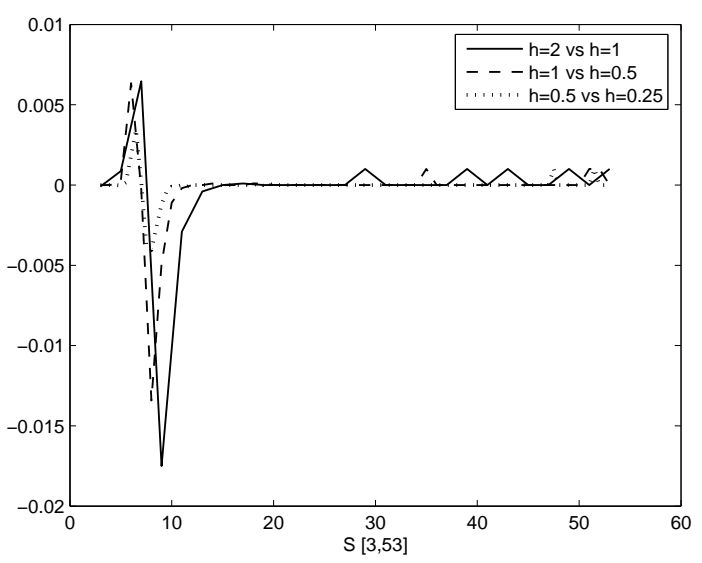

Fig. 2. Different spatial semidiscretizations with Dividend Type A

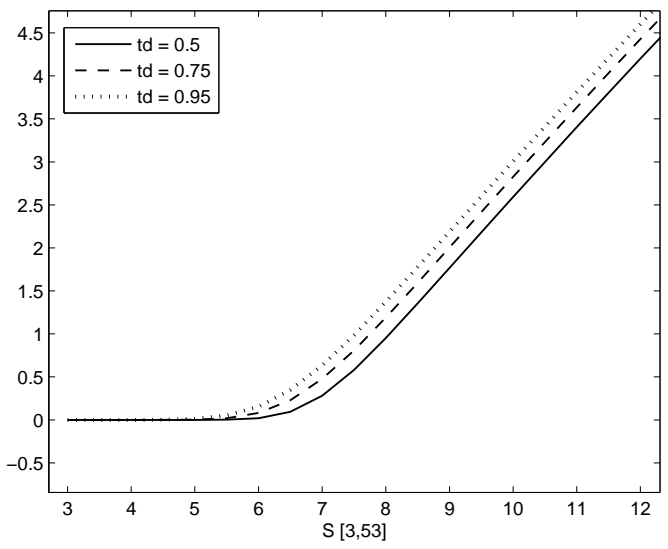

Fig. 3. Different dividend dates

Let us denote the numerical valuation of the call option at $t=0$ by $C(S, t=0, h)$, when a step size $h$ is used. In order to estimate the stability of the method, we show the difference $C(S, t=0, h / 2)-C(S, t=0, h)$, for various values of $h$ for the data of this example, see figure 2 .

Figure 3 shows the valuation of the call option for different dividend payment dates. Finally, figure 4 shows the valuation of the call option with strike price $E=27$ for various values of the volatility.

EXAMPLE 3. Let us consider the valuation problem of a vanilla call option modeled by (3)-(4), where

$$
D_{\delta}(S)=A S^{3}
$$




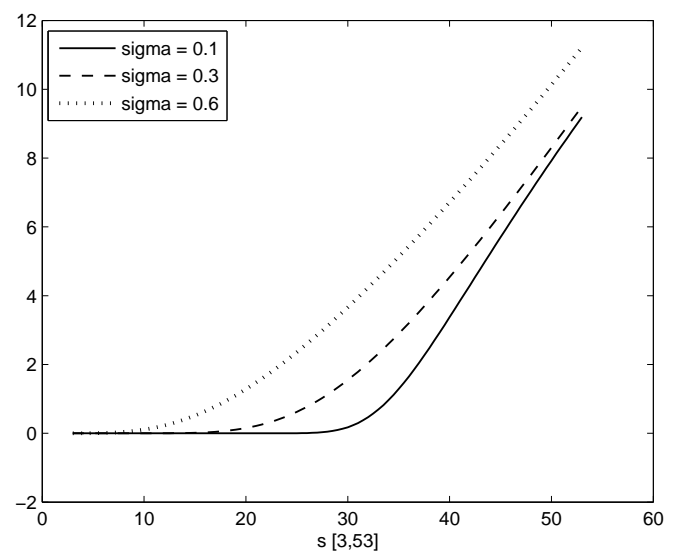

Fig. 4. Different volatilities

and

$$
f(S)=\max \{S-E, 0\}, \quad 0<S<\infty .
$$

For $A=0.001, \sigma=0.1, r=0.08, T=1, t_{d}=0.5, E=7, S_{0}=3, N=100$ and $h=0.5$, by (43) one gets the numerical solution $v(0)$, see the dotted line in figure 1 , called dividend type $B$.

\section{References}

[1] M. Avellaneda and P. Laurence, Quantitative Modeling of Derivative Securities, Chapman \& Hall / CRC, London, 2000.

[2] R. Company, A. L. González and L. Jódar, Numerical solution of modified Black-Scholes equation pricing stock options with discrete dividend, Mathematical and Computer Modelling 44 (2006), 1058-1068.

[3] T. M. Flett, Differential Analysis, Cambridge Univ. Press, 1980.

[4] I. Higueras and B. García-Celayeta, Logarithmic norm of a matrix pencil, SIAM J. Matrix Anal., 20 (3), (1999), 646-666.

[5] G. E. Shilov, Generalized Functions and Partial Differential Equations, Gordon and Breach, 1968.

[6] G. D. Smith, Numerical Solution of Partial Difference Equations: Finite Difference Methods, Oxford Univ. Press, Third Edition, Oxford, 1985.

[7] P. Wilmot, S. Howison and J. Dewyne, The Mathematics of Financial Derivatives, Cambridge Univ. Press, Cambridge, 1995. 
